\title{
METAFÍSICA, LÓGICA Y MUNDO \\ UNA MIRADA ANTROPOLÓGICA DE \\ LOS CONCEPTOS FUNDAMENTALES DE LA METAFISICA. Mundo, Finitud, Soledad, 1929/30, de Martin HeidegGeR ${ }^{1}$
}

Enrique V. Muñoz Pérez Universidad Católica del Maule enmunoz@ucm.cl

\begin{abstract}
Resumen / Abstract
Este artículo pretende ser una interpretación de algunos pasajes de la lección Los conceptos fundamentales de la metafisica. Mundo, finitud, soledad que Martin Heidegger dictara en la Universidad de Friburgo (Alemania), el semestre de invierno de 1929/30. Mi hipótesis investigativa es que Heidegger lleva a cabo una lectura metafísica de la lógica, cuyas consecuencias son antropológicas, en el sentido que permiten establecer la distinción entre el animal y el ser humano. En otras palabras, me parece que el detenido análisis sobre el "como" (als) es, en parte, la respuesta heideggeriana a una de las preguntas antropológicas fundamentales de la época, esto es: ¿dónde radica la diferencia entre el animal y el ser humano?
\end{abstract}

Palabras Clave: ser humano, animal, lógica, mundo, metafísica, como.

\section{METAPHYSICS, LOGIC AND WORLD. AN ANTHROPOLOGICAL LOOK AT THE FUNDAMENTAL CONCEPTS OF METAPHYSICS. WORLD, FINITUDE, SOLITUDE 1929-1930 BY MARTIN HEIDEGGER}

This article offers an interpretation of certain passages of the lectures on The Fundamental Concepts of Metaphysics: World, Finitude, Solitude which Martin Heidegger dictated at the University of Freiburg (Germany), in the winter semester of 1929/30. My research hypothesis is that Heidegger provides a metaphysical reading of logic that have anthropological consequences in the sense that they allow the distinction between animals and humans. In other words, I think the detailed analysis on the "as" (als) is, in part,

Este artículo es una versión ampliada y corregida de una ponencia que desarrollé en el I Congreso de la Sociedad Iberoamericana de Estudios Heideggerianos, desarrollada en conjunto por la Facultad de Filosofía de la P. Universidad Católica de Chile y el Departamento de Filosofía de la Universidad Alberto Hurtado, los días 26, 27 y 28 de septiembre de 2011 en Santiago, Chile. Este trabajo también forma parte del Proyecto de Investigación de Iniciación sobre el tema “ ¿Ser humano o animalidad?: Tarea y alcances de Los conceptos fundamentales de la metafisica. Mundo-finitud-soledad de Martin Heidegger" (2011-2013), que cuenta con apoyo financiero del Fondecyt (Chile) (Proyecto No 11110127). Agradezco al profesor Dr. César Lambert por la discusión inicial de este artículo y al profesor Dr. Alejandro G. Vigo por sus observaciones y comentarios que han enriquecido la versión final de este texto. 
the Heideggerian response to one of the fundamental anthropological questions of the time, that is: what is the difference between an animal and a human being?

KEY WORDS: Human being, animal, logic, world, metaphysics, as.

\section{Introducción}

$\widehat{R A}$

Mi interés principal en relación con la lección magistral Los conceptos fundamentales de la metafísica. Mundo, finitud, soledad ${ }^{2}$, que corresponde al curso que Heidegger dictara el semestre de invierno de 1929/30 en la Universidad de Friburgo, ha sido desde hace un tiempo su dimensión temática: esto es, el novedoso tratamiento de la cuestión del mundo desde la conocida comparación entre la piedra, el animal y el ser humano ${ }^{3}$. Particularmente interesante me ha parecido, la caracterización del ser humano como formador de mundo ("Der Mensch als weltbildend"), lo que constituye una novedad, si se tiene presente el abandono del concepto "ser humano" (Mensch) en Ser y Tiempo ${ }^{4}$.

Sin embargo, no pretendo quedarme exclusivamente en la mencionada dimensión temática de Los conceptos fundamentales de la metafisica. Mundo, finitud, soledad, sino que dar un paso más y relacionar esta discusión sobre el concepto de "mundo" y el ser humano con el surgimiento de cuestiones lógicas al final de la mencionada lección. Me refiero, específicamente, al aparente "cambio" en la estructura argumental que se produce en los parágrafos 69 a 72 .

Como ya sostuve, dado el énfasis de mi investigación en la dimensión temática, me parecía extraño que Heidegger se concentrara en cuestiones como la interpretación formal sobre el "en tanto que" o "como" (als) $)^{5}$, la reflexión metódica fundamental o la caracterización de la proposición enunciativa, cuando se había dedicado extensamente, de manera previa, a pensar la animalidad entre los parágrafos 45 a 61. En otras palabras, las preguntas que me surgían eran: ¿por qué aparece esta discusión sobre la dimensión lógica en la lección Los conceptos fundamentales de la metafísica. Mundo, finitud, soledad? y ¿cómo se relaciona esta discusión sobre la lógica con la aproximación temática?

Una posible explicación es la siguiente: El desarrollo que hace Heidegger entre los parágrafos 69 a 72 de Los conceptos fundamentales de la metafísica. Mundo, finitud,

2 En el artículo citaré, en primer lugar, la traducción al español y, posteriormente, el original en alemán separado por /.

Muñoz, Enrique 2008, pp. 121 ss.

Muñoz, Enrique 2007, pp. 91-105.

Voy a entender el término alemán "als" indistintamente como "como" y como "en tanto que". Esta última es la opción que utiliza el traductor de Los conceptos fundamentales de la metafisica. Mundo, finitud, soledad y también el traductor de Ser y Tiempo. 
soledad no corresponde estrictamente a lo que los especialistas de la filosofía del lenguaje denominarían como "metalógica" o "metalenguaje"; sino que, más bien, Heidegger lleva a cabo una lectura metafísica de la lógica cuyas consecuencias, a mi modo de ver, son antropológicas, en el sentido que permiten establecer la distinción entre el animal y el ser humano. En otras palabras, me parece que el detenido análisis sobre el "como" (als) o sobre el enunciado apofántico, es, en parte, la respuesta heideggeriana a una de las preguntas antropológicas fundamentales de la época -finales de los años veinte en Alemania-, esto es: ¿dónde radica la diferencia entre el animal y el ser humano?

Es conocido que en el año 1928, Max Scheler publica el libro El puesto del hombre en el cosmos. Allí Scheler lleva a cabo una comparación entre la planta, el animal y el ser humano estableciendo que hay grados del ser psicofísicos que estos tres seres vivos comparten (impulso afectivo, instinto, memoria asociativa e inteligencia práctica); sin embargo, la diferencia esencial entre el animal y el ser humano se encuentra en lo que Scheler llama el espíritu. Estos planteamientos constituyen parte del contexto en el que se desarrolla la lección Los conceptos fundamentales de la metafísica. Mundo, finitud, soledad ${ }^{6}$ y que llevan, a mi juicio, a Heidegger a tomar distancia de los análisis biológicos y científicos y a confiar en los alcances de su proyecto filosófico, por esos días de impronta metafísica, para establecer la diferencia entre el animal y el ser humano. Dicho en otros términos, para Heidegger el ser humano tiene la posibilidad de representarse el mundo "como" (als) un todo de significatividad o "como" (als) manifestabilidad de lo ente en cuanto tal en su conjunto, cuestión que al animal le está negada. Denominaré a esta explicación, hipótesis diferenciadora entre el animal y el ser humano.

Me abocaré, a continuación, a la aclaración de esta hipótesis. Para ello, dividiré mi artículo en tres momentos: I. Breves antecedentes de la discusión lógica en el pensamiento de Heidegger; II. Una mirada antropológica a la interpretación formal sobre el "como" (als) y III. Palabras finales.

\section{Breves antecedentes de la discusión lógica en el pensamiento de Heidegger}

Los antecedentes de la discusión lógica que desarrolla Heidegger en Los conceptos fundamentales de la metafísica. Mundo, finitud, soledad son precisados por él mismo en el parágrafo 72 de la mencionada lección. Heidegger los entiende como estaciones

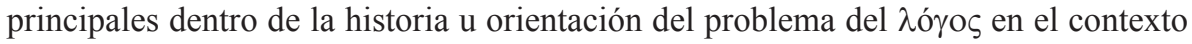
de la metafísica, pero que, a su juicio, no dan ninguna orientación completa sobre el

$6 \quad$ Así lo establece Heidegger mismo:'Recientemente, en el contexto de una antropología, Max Scheler ha tratado de tratar unitariamente esta gradación del ente material, la vida y el espíritu, partiendo de un convencimiento según el cual el hombre es el ser que unifica en sí mismo todos los niveles de lo ente: el ser físico, el ser de la planta y del animal, y el ser específicamente espiritual" (Heidegger 2007, p. 242 / 1992, p. 283). 
desarrollo de la problemática misma. Dichos hitos son los siguientes: "Ser y Tiempo, $\S 7 \mathrm{~B}, \S 33$ y $\S 44 ;$ Kant y el problema de la metafisica, $\S 7, \S 11$ y todo el tercer apartado del libro; De la esencia del fundamento, Primer apartado, el problema del $\lambda$ óyo $\varsigma$ en Leibniz en relación con la pregunta metafísica por el ser" (Heidegger 2007, p. 365 / 1992, p. 441).

En el marco de estos hitos, focalizaré mi interés en las siguientes temáticas: la interpretación heideggeriana del logos apofántico, las referencias al "como" (als) como estructura lógica y la relación con el tema que desarrollo.

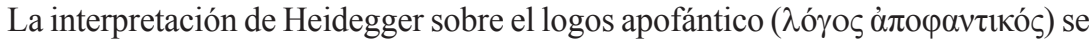
encuentra principalmente en Ser y Tiempo; en dicha obra, Heidegger se distancia de la interpretación tradicional del $\lambda$ ó $\gamma$ o $\varsigma$ de la verdad como "concordancia" o "adecuación"

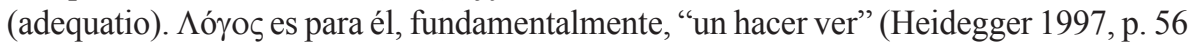
/ 2001, p. 32). Por ello, el ser verdadero del $\lambda$ ó $\gamma$ o $\varsigma$ quiere decir "sacar de su ocultamiento el ente del que se habla, y hacerlo ver como desoculto, es decir, descubrirlo" (Heidegger, 1997, p. 56 / 2001, p. 33) Como es sabido, Heidegger bosqueja aquí su interpretación de la verdad como à $\lambda \hat{\theta} \theta \varepsilon ı \alpha$ que desarrollará en el parágrafo 44 de Ser y Tiempo.

Con todo, la interpretación de Heidegger no se reduce únicamente a sostener la mencionada caracterización del $\lambda$ ó $\gamma o \zeta$, sino que se extiende a otros sentidos tradicionales del término. De este modo, Heidegger sostiene que $\lambda o ́ \gamma o \varsigma$ hace referencia también al fundamento o la razón der ser y a la relacionalidad. El $\lambda$ ó $\gamma o \varsigma$ es tanto "fundamento de toda posible interpelación y discusión" como "aquello que se ha hecho visible en su relación con algo”. (Heidegger 1997, p. 57 / 2001, p. 34) Más allá de las aproximaciones filológicas a estos fragmentos sobre el $\lambda$ ó $\gamma \circ \varsigma$, me interesa resaltar que Heidegger busca superar la comprensión clásica del logos apofántico tratando de hacer ver que es necesario pensar de una manera distinta la relación entre la lógica y la ontología -la metafísica en el lenguaje de Los conceptos fundamentales de la metafísica. Mundo, finitud, soledad. Previo al logos apofántico, hay que tener presente un logos existencial o hermenéutico, que dé cuenta de la profundidad de la existencia humana. Es decir, la lógica y sus enunciados no pueden ser comprendidos únicamente como el vestíbulo del conocimiento 7 . Más aún, según Heidegger el enunciado lógico no puede negar su origen ontológico en la interpretación comprensora.

$7 \quad$ Esta manera de entender la lógica no es nueva en el pensamiento de Heidegger, puesto que se puede rastrear una idea similar en las Interpretaciones fenomenológicas sobre Aristóteles. Allí Heidegger afirma que las mencionadas interpretaciones son una contribución a la historia de la ontología y de la lógica. "La idea de la hermenéutica fenomenológica y de la facticidad incluye entre sus tareas: la teoría formal y material del objeto de la lógica, la doctrina de la ciencia, la "lógica de la filosofía", la "lógica del corazón", la lógica del pensamiento "preteorético y práctico"; e incluye todas esas tareas no en el sentido de un concepto colectivo, sino en el sentido de que - a tenor de su eficacia-proporciona el punto de partida fundamental de la problemática filosófica" (Heidegger 2002, p. 48 / 2005, p. 365). 
El "en cuanto" (als) originario de la interpretación circunspectivamente comprensora será llamado "en cuanto" (als) hermenéutico-existencial, a diferencia del "en cuanto" (als) apofántico del enunciado (Heidegger 1997 p. 181 / 2001, p. 158. El subrayado es mío).

Para esclarecer esta afirmación, me parece necesario abordar algunos aspectos del parágrafo 32 de la obra fundamental de Heidegger. La discusión del mencionado parágrafo se orienta a esclarecer la relación entre comprensión e interpretación. El punto que me interesa subrayar es el siguiente: Heidegger sostiene que lo explícitamente comprendido "tiene la estructura del algo en cuanto algo [etwas als etwas]" (Heidegger 1997, p. 172 / 2001, p. 149). Para Heidegger, entonces, lo abierto en el comprender, lo comprendido, es accesible de manera tal que en él se puede destacar explícitamente su "en cuanto que" [als was] (Heidegger 1997, p. 173 / 2001, p. 149). En otras palabras, cuando el ente intramundano ha sido descubierto por medio del ser del Dasein, es decir, "cuando ha venido a comprensión [zu Verständnis gekommen ist], decimos que tiene sentido" (Heidegger 1997, p. 175 / 2001, p. 151. El subrayado es mío).

Sobrepasa el objetivo del presente artículo clarificar el muy transitado problema del sentido en el pensamiento de Heidegger. Me interesa únicamente enfatizar que solo el Dasein "tiene" sentido, "en la medida en que la aperturidad del estar-en-el-mundo puede ser "llenada" por el ente en ella descubrible" (Heidegger 1997, p. 175 / 2001, p. 151). En consecuencia, todo ente que tenga un modo de ser diferente del modo de ser del Dasein, tales como el martillo o el animal, "deberá ser concebido como sin sentido, como esencial y absolutamente desprovisto de sentido" (Heidegger 1997, p. 175 / 2001, p. 152. El subrayado es mío). Considero, entonces, que la distinción entre existencia humana (Dasein) con sentido y los otros entes (por ejemplo, el animal) sin sentido, es un antecedente relevante para la distinción que se desplegará en la lección Los conceptos fundamentales de la metafísica. Mundo, finitud, soledad.

No obstante lo anterior, hay un par de antecedentes menores que complementan los pasajes de Ser y Tiempo, respecto de nuestra hipótesis de trabajo. La primera se encuentra en De la esencia del fundamento (1929) y la segunda en la lección sobre Leibniz, conocida como Principios metafísicos de la lógica (1928). En el primer caso, Heidegger hace presente que la verdad de lo que está ahí delante (el objeto, la piedra, la planta o el animal) es distinta de la verdad del Dasein. De paso, Heidegger vuelve sobre la distinción entre verdad de la proposición y verdad más originaria ${ }^{8}$. El segundo caso, es el más evidente, puesto que la afirmación de Heidegger es lapidaria: juzgar

8 "Para ser posible, la predicación tiene que poder asentarse en un hacer-manifiesto que no tiene carácter predicativo. La verdad de la proposición hunde sus raíces en una verdad más originaria (desocultamiento), esto es, en el carácter manifiesto antepredicativo de lo ente, que recibe el nombre de verdad óntica. Su posible carácter manifiesto y los correspondientes modos del determinar explicativo e interpretativo se transforman de acuerdo con los

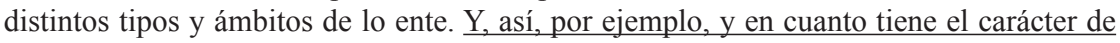
algo descubierto, la verdad de lo que está ahí delante (por ejemplo, las cosas materiales) se 
es un comportamiento del hombre. El contexto de las expresiones heideggerianas está constituido por el esclarecimiento de la relación entre lógica y ontología. Heidegger se pregunta, entonces, cómo debe plantearse y ser tratada una teoría sobre la esencia del juicio. Su respuesta es la siguiente: "Juzgar es un comportamiento del hombre en tanto que juzgar sobre el ente, el juzgar sobre ... está relacionado con el ente" (Heidegger 2009, p. 119 /1990, p. 124. El subrayado es mío).

Aportados estos antecedentes, me abocaré a continuación a desarrollar una aproximación antropológica a la interpretación formal sobre el "como" (als).

\section{Una mirada antropológica a la interpretación formal sobre el "como" (als)}

Las tres tesis a partir de las cuales Heidegger busca aproximarse al fenómeno del mundo son bien conocidas: "1) la piedra (lo material) es sin mundo; 2) el animal es pobre de mundo; 3) el ser humano configura [o forma] mundo. [1.der Stein (das Materielle) ist weltlos; 2. das Tier ist weltarm; 3. der Mensch ist weltbildend]" (Heidegger 2007, p. 227 / 1992, p. 263). Heidegger formula esta tres tesis en la búsqueda de una nueva aproximación al fenómeno del mundo que no sea la de Ser y Tiempo ni la De la esencia del fundamento, esto es, que no sea una interpretación del modo como nos movemos y nos relacionamos cotidianamente con los entes intramundanos, ni un tratamiento histórico sobre el concepto de mundo. La opción heideggeriana, esta vez, es una consideración comparativa entre las tres tesis antes mencionadas. Ello lleva a Heidegger a concentrarse largamente en la tesis intermedia sobre el animal y esclarecer, de paso, nociones como la de órgano, organismo y la esencia de la animalidad. En el fondo, Heidegger busca establecer algunos puntos de diferenciación entre el ser humano y el animal.

El criterio fundamental para distinguir entre el ser humano y el animal se encuentra en que el ser humano forma o configura mundo. El animal, en cambio, solo tiene un acceso limitado al mundo o a lo ente. Heidegger lo ejemplifica de la siguiente manera: "El nido que se busca, la presa que se caza, después de todo no son una nada, sino que un ente, de otro modo el pájaro no se podría posar en el nido ni el gato cazar un ratón, si no fueran ente" (Heidegger 2007, p. 324 / 1992, p. 390).

Es claro que el animal tiene un acceso concreto a algo que realmente es, "sin embargo, eso es algo que solo nosotros somos capaces de experimentar y, ostensiblemente, de tener, en tanto que ente. [Das Tier hat einen Zugang zu ..., und zwar zu solchem, was wirklich ist - was aber nur wir als Seiendes zu erfahren und offenbar zu haben vermögen]" (Heidegger 2007, 324/ 1992, 390. El subrayado es mío). Por ello es que en el animal, afirma Heidegger, hay un tener y un no tener mundo.

distingue específicamente de la verdad de lo ente que somos nosotros mismos, del carácter de apertura del Dasein existente" (Heidegger 2000, p. 115 /1996, pp. 130-1). 
Me restan todavía algunos pasos para abordar el título de este apartado, me refiero a la explicación que hace Heidegger del giro "formación o configuración de mundo".

Que el ser humano qua ser humano sea configurador de mundo no significa el ser humano tal como va andando por la calle, sino que el ser-ahí en el ser humano es configurador de mundo [das Da-sein im Menschen ist weltbildend] (Heidegger 2007, p. 344 / 1992, p. 390. El subrayado es mío).

¿Qué quiere decir “configuración de mundo”? Heidegger aclara que esta expresión es polisémica. Significa "producir" el mundo, "dar una imagen" suya, "representárselo", abarcarlo, etc. En otras palabras, el ser humano no solo produce artefectos o construye puentes, sino que forma con otros seres humanos su historia, su tiempo y su época.

Dicho esto, y haciendo presente que aún le resta interpretar más precisamente el fenómeno del mundo, Heidegger desarrolla inmediatamente la primera interpretación formal del "como" o "en tanto que" (als) como un momento estructural de la manifestabilidad del mundo. Heidegger aborda, de este modo, la conexión del "en tanto que" (als) como la estructura de la relación y de los miembros de la relación con la proposición enunciativa.

Dos son, a mi juicio, los pasajes relevantes que me permiten sostener que este tratamiento del "en tanto que" o del "como" (als) constituye uno de los criterios, en este caso lógico, para diferenciar entre el ser humano y el animal.

Heidegger comienza, en primer lugar, recapitulando lo expuesto. Se ha dicho que mundo es la manifestabilidad de lo ente en cuanto tal en su conjunto. Con esta caracterización emerge algo enigmático: este "en cuanto tal", lo ente en cuanto tal, algo en tanto que algo. Y he aquí lo relevante:

este "en tanto que", que es totalmente elemental, es -así podemos decirlo simplemente- 1 lo que le es negado al animal. [Dieses ganz elementare "als" ist es -so können wir ganz einfach sagen- was dem Tiere vesagt ist] (Heidegger 2007, p. 346 / 1992, p. 416. El subrayado es mío).

En otras palabras, el "en tanto que" o "como" (als) marca la diferencia entre lo animal y lo humano. El animal no puede representarse la "piedra como martillo" o tomar su vida "como un todo", al modo como lo hace el ser humano.

El "en tanto que" o "como" (als) está conectado, agrega Heidegger, en segundo lugar, con el problema conductor del mundo que está formulado en la tesis: el ser humano es configurador de mundo. Heidegger hace presente que no le interesa partir de una definición arbitraria y dada del ser humano, sino que busca, al menos, cuestionarla. Enfatiza, además, que desarrolla el problema del mundo desde un temple de ánimo fundamental: el aburrimiento. Con todo, cuando se aboca a aclarar el momento estructural del "en tanto que algo", Heidegger se percata de que esta tarea no es tan evidente. Normalmente creemos que el "en tanto que" (als) es una relación. Sin embargo, esto que parece obvio, esconde un problema: 
La base de la metafísica que hemos caracterizado, y su orientación con arreglo a la verdad de la proposición, aunque en cierto sentido es necesaria, sin embargo, no es original (Heidegger 2007, p. 349 / 1992, pp. 420-1).

Esta no-originalidad ha impedido, sostiene Heidegger, el correcto desarrollo del problema del mundo. En otras palabras, se hace necesario esclarecer la relación entre metafísica y lógica. Hay que desarrollar, con palabras del Informe Natorp, una "lógica del pensamiento "preteórico y práctico" (Heidegger 2002, 48/ 2005, 365) que proporcione el punto de partida fundamental de la problemática metafísica u ontológica.

No obstante lo anterior, hay que esperar hasta la caracterización de la proposición enunciativa en el parágrafo 72 de Los conceptos fundamentales de la metafísica. Mundo, finitud, soledad para sellar la diferencia entre el ser humano y el animal. Como es sabido, Heidegger cita a Aristóteles sosteniendo que $\lambda$ ó $\gamma o \zeta$ significa el discurso, el conjunto de lo hablado y de lo decible. Por otro lado, "lenguaje" alude a la capacidad fundamental de hacer discurso y, en consecuencia, del hablar. "Así los

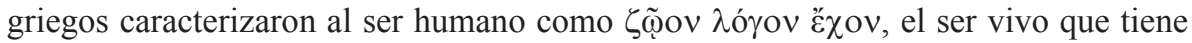
como posesión esencial la posibilidad del discurso. El animal es, en calidad de ser vivo

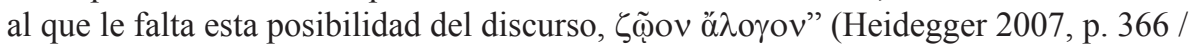
2005 , p. 442). Además, al animal le falta percibir como un ente aquello a lo cual está abierto y también, como veremos, percibir el mundo en su conjunto.

Heidegger reitera más adelante el valor de la estructura del "en tanto que" (als) en la diferenciación entre el ser humano y el animal: en el primero hay comportamiento, en segundo caso conducta. En otras palabras, forma parte del ser humano

un estar abierto a ... de tal tipo que este estar abierto a ... tiene el carácter de percibir algo en tanto que algo [Vernehmens von etwas als etwas]. A este tipo del referirse a lo ente lo llamamos comportamiento [Verhalten], a diferencia de

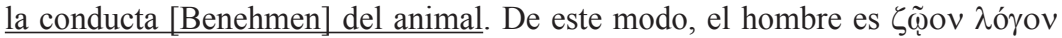

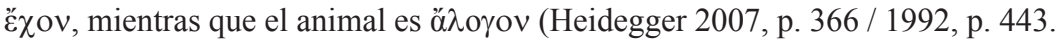
El subrayado es mío).

En definitiva, el ser humano tiene la capacidad de hablar, de conversar, de pedir, de desear, de preguntar, de narrar, etc., que constituyen la comprensibilidad [Verständlichkeit], cuestión que, hasta donde se sabe, le está impedida al animal.

No obstante lo anterior, naturalmente, la reflexión heideggeriana no se queda en una mera reinterpretación del pensamiento de Aristóteles. Heidegger enfatiza que no hemos alcanzado aún el planteamiento correcto y que, todavía más, dicho planteamiento está ausente en la reflexión aristotélica sobre el $\lambda$ óyos; él persigue demostrar, en último término, su fundamento último.

A juicio de Heidegger, este fundamento radica en la libertad humana. El $\lambda$ ójos, en la forma del $\lambda o ́ \gamma o \varsigma \dot{\alpha} \pi \circ \varphi \alpha v \tau$ ikós, es la potencia de un comportamiento que muestra lo ente, ya sea descubriendo (verdadero) u ocultando (falso). "Esta potencia es como potencia solo posible, si se fundamenta en un ser libre para lo ente en cuanto tal [Freisein für das Seiende als solches]" (Heidegger 2007, p. 403 /1992, p. 492). En dos palabras, 


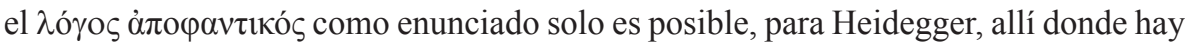
libertad. Y este comportamiento libre del ser humano tiene su expresión privilegiada en la tercera tesis heideggeriana: el ser humano es formador o configurador de mundo. "Los juicios y las proposiciones no son en sí mismos primariamente configuradores de mundo, aunque pertenecen a la configuración del mundo" (Heidegger 2007, p. 405 / 1992, p. 496. El subrayado es mío). Antes de cualquier descubrimiento u ocultamiento asociado al $\lambda$ ó $\gamma$ $\varsigma$, tiene que ser posible, en el ser humano que enuncia, un previo estar abierto al ente mismo sobre el que se juzga en cada caso. De este modo, el regreso a

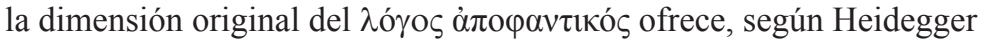

un rico plexo estructural [Strukturzusammenhang], articulado en sí mismo, que
ostensiblemente caracteriza un acontecer fundamental en la existencia del hombre
[Grundgeschehen im Dasein des Menschen], que fijamos con un triple momento:
1) el tenderse a la vinculatoriedad [das Entgegenhalten der Verbindlichkeit]; 2)
el completamiento [die Ergänzung]; 3) el descubrimiento del ser de lo ente [die
Enthüllung des Seins des Seienden] (Heidegger 2007, p. 413/ 1992, p. 506. El
subrayado es mío).

Para comprender mejor este pasaje, me parece necesario hacer una breve síntesis de las principales tesis de Heidegger hasta el momento: Él ha sostenido en la lección Los conceptos fundamentales de la metafisica. Mundo, finitud, soledad que entiende el mundo como la manifestabilidad de lo ente "en cuanto tal" (als) en su conjunto. El sentido de la pregunta por el "como" o "en cuanto tal" (als) dice relación, por consiguiente, con abordar desde allí el fenómeno del mundo. Eso lo lleva a concluir, como he intentado mostrar, que el "como" o "en cuanto tal" (als) es "algo distintivo en aquello a lo cual está abierta la existencia humana, a diferencia del estar abierto a... en el caso del animal". [Das "als" ist etwas Auszeichnendes an dem, wofür das menschliche Dasein offen ist, im Unterschied zu dem Offensein für... beim Tier]" (Heidegger 2007, p. 396 / 1992, p. 482. El subrayado es mío). En el caso del animal el "estar abierto a" es, más bien, un estar absorbido por el mundo. El animal queda preso de su entorno y no puede ir más allá; por eso, no modela mundos.

En cambio, en el caso del ser humano, este "como" (als) nos acerca a su esencia. Dicho de otra manera, Heidegger pretende que los análisis de la estructura o fundamento de la posibilidad interna del $\lambda$ ó $\gamma$ o del ser humano en su época. No hay que dejar de lado que Heidegger ha comenzado la lección sobre Los conceptos fundamentales de la metafísica. Mundo, soledad, finitud haciendo referencia a que el ser humano de fines de la década de los años veinte del siglo pasado es víctima del aburrimiento.

Heidegger, entonces, afina sus análisis tratando de mostrar cuál es la relación entre estos estudios sobre el $\lambda$ ó $\gamma o \zeta$, el ser humano y la tesis de la configuración del mundo. Uno de los indicadores que provienen de este análisis es la, ya citada, "vinculatoriedad" (Verbindlichkeit). En términos sencillos, el ser humano mediante el uso de su $\lambda$ ó $\gamma o \varsigma$ se vincula con o se abre a lo ente. El $\lambda$ ó $\gamma o \zeta$ tiene la potencia y la posibilidad, fundada en la libertad del ser humano, de sostener que un ente es verdadero o falso, es decir, tiene la posibilidad de asignar el desencubrimiento o el ocultamiento del ente. Esta capacidad 
de vincularse con el ente no es una cuestión añadida, sino que forma parte de sí. Se va clarificando, paulatinamente, la diversidad esencial entre el estar abierto del animal y la apertura al mundo del hombre. "Estar abierto del hombre es estar ofrecido a, estar abierto del animal es estar absorbido por..., y, en ello, estar cautivado en el anillo" (Heidegger 2007, p. 407 / 1992, p. 498. El subrayado es mío).

Un segundo indicador, está constituido por el "completamiento" (Ergänzung). El ser humano se relaciona con los entes en su conjunto o de modo completo. El ejemplo de Heidegger tiene que ver con la ubicación de una pizarra en la sala de clases, que normalmente debe estar detrás del profesor. Heidegger se pregunta por qué nos parece extraño que dicha pizarra se encuentre en una esquina; por qué decimos que la "pizarra está mal ubicada". Para él, como ya se dijo, nos relacionamos con el todo que denominamos "sala de clases". Este conjunto constituye el entorno en el que nos movemos o en el que nos encontramos habitualmente. Precisamente "donde nos movemos ya desde siempre es lo que designamos en primer lugar esquemáticamente como el "en su conjunto" [im Ganzen]" (Heidegger 2007, p. 412 / 1992, p. 505). Siguiendo el ejemplo de Heidegger, por qué nos incomoda que los libros de la biblioteca de la universidad estén en el suelo; porque ellos habitualmente se encuentran en los "estantes".

En consecuencia, a partir de la "vinculatoriedad" y del "completamiento" es posible para el ser humano pronunciarse sobre el ente, "es decir, decir del ser-qué; del ser-asi, del ser-que y del ser verdad [d.h. vom Wassein, Sosein, Daßsein und Wahrsein]" (Heidegger 2007, p. 413 / 1992, p. 506).

Con estos tres momentos - la vinculatoriedad, el completamiento y el descubrimiento del ser de lo ente- se caracteriza, según Heidegger, un acontecer fundamental unitario en la existencia del ser humano, del cual surge el $\lambda$ ó $\gamma$ os.

\section{Palabras finales}

El objetivo del presente artículo era esclarecer las siguientes preguntas: ¿por qué aparece la discusión sobre la dimensión lógica en la lección Los conceptos fundamentales de la metafísica. Mundo, finitud, soledad? y ¿cómo se relaciona esta discusión sobre la lógica con la discusión temática propuesta en la lección? Al respecto, quisiera afirmar lo siguiente:

1. Me parece que el tratamiento de cuestiones lógicas, tales como el "en tanto que" o "como" (als) y el "enunciado apofántico" al final de la mencionada lección, puede ser entendido como la respuesta metafísico-lógica de Heidegger a la pregunta por la diferencia entre lo animal y lo humano. En otras palabras, Heidegger no sigue el modelo de Scheler o de Plessner, quienes buscan ya sea en las diferencias psicofísicas o en la biología la respuesta a la distinción entre el ser humano y el animal. Él pone su énfasis en la libertad humana que, mediante su comportamiento fundado en el logos, se vincula con los entes, los comprende en su conjunto, reflexiona sobre ellos, etc.; esto significa, en último término, que el ser humano configura o modela su mundo. Si lo anterior es 
correcto, es posible entender el sentido antropológico de los prolijos y técnicos estudios de Heidegger acerca de la lógica, el lenguaje, el enunciado apofántico o la indicación formal.

2. No hay, entonces, como me parecía ver inicialmente, un quiebre en la argumentación heideggeriana entre la discusión temática sobre el mundo y las observaciones acerca de la lógica. Mi sospecha queda refrendada en el siguiente pasaje de Los conceptos fundamentales de la metafisica. Mundo, finitud, soledad con el que concluyo:

Si $\lambda$ ójo representa en la Antigüedad el fenómeno a partir del cual se comprende al ser humano en lo que tiene de propio, y si nosotros mismos decimos que la esencia del ser humano es configuradora de mundo, entonces en ello se expresa que, si ambas tesis guardan alguna conexión en general, entonces, $\lambda$ ó $\gamma o$ s, lenguaje y mundo guardan una relación interna (Heidegger 2007, p. 366 / 1992, p. 443. El subrayado es mío).

\section{Referencias bibliográficas}

Heidegger, Martin (1990), Metaphysische Anfangsgründe der Logik im Ausgang von Leibniz, editado por Klaus Held, GA 26. Frankfurt a.M.: Editorial Klostermann. (1992), Die Grundbegriffe der Metaphysik, editado por FriedrichWilhelm von Herrmann, GA 29/30. Frankfurt a.M.: Editorial Klostermann.

(1996), Wegmarken, editado por Friedrich-Wilhelm von Herrmann.

Frankfurt a.M.: Editorial Klostermann

(1997), Ser y Tiempo, traducido por Jorge E. Rivera. Santiago de Chile: Editorial Universitaria.

(2000), Hitos, traducido por Helena Cortés. Madrid: Alianza Editorial. (2001), Sein und Zeit. Tübingen: Editorial Max Niemeyer.

(2002), Interpretaciones fenomenológicas sobre Aristóteles, traducido por Jesús A. Escudero. Madrid: Editorial Trotta.

(2005), Phänomenologische Interpretationen ausgewählter Abhandlungen des Aristoteles zur Ontologie und Logik, editado por Günther Neumann, GA 62. Frankfurt a.M.: Editorial Klostermann.

(2007), Los conceptos fundamentales de la metafísica. Mundo, finitud, soledad, traducido por Alberto Ciria. Madrid: Alianza Editorial.

(2009), Principios metafísicos de la lógica, traducido por Juan José Garcia Norro. Madrid: Editorial Síntesis.

Muñoz, Enrique (2008), Der Mensch als Zentrum, aber nicht als Mensch. Zur Konzeption des Menschen in der ontologischen Perspektive Martin Heideggers, Würzbug: Editorial Ergon.

(2007), "Heidegger y la pregunta por el ser humano", Revista Veritas, Vol. II, No 16, Marzo: 91-105. 